\title{
CONSTRUINDO UM HISTÓRICO DE ENFERMAGEM PARA SER UTILIZADO COM O IDOSO INTERNADO EM UM HOSPITAL UNIVERSITÁRIO
}

Fernanda Lucas Lopes ${ }^{1}$, Silvana Sidney Costa Santos ${ }^{2}$

RESUMO: Estudo metodológico objetivando construir instrumento de coleta de dados para o idoso hospitalizado. Desenvolveu-se em três fases: 1) construção do instrumento; 2) validação do conteúdo do instrumento; 3) testagem clínica com idosos. Na primeira fase foram realizadas revisão de literatura e construção da versão inicial do instrumento, levando à identificação de 525 sinais e sintomas, ordenados numa Escala Likert. A segunda fase realizou-se em duas etapas: 1) versão inicial do instrumento encaminhado a 21 enfermeiras, para validação de conteúdo dos itens, identificandose 223 itens que apresentaram a média ponderada $\geq a$ 80\%; 2) construção definitiva do instrumento, constituído de sete partes. Na última fase foi testado o instrumento final em dez idosos. Espera-se que sua utilização contribua para que as enfermeiras possam orientar o idoso, a família, o cuidador e a equipe de enfermagem, no atendimento das necessidades afetadas e na minimização das dificuldades instaladas.

PALAVRAS-CHAVE: Idoso; Estudos de validação; Pesquisa metodológica em enfermagem.

\section{THE DEVELOPMENT OF A NURSING HISTORIC TO BE USED WITH HOSPITALIZED ELDERLY IN A UNIVERSITY HOSPITAL}

ABSTRACT: Methodological research which objective is to prepare a data collection instrument for hospitalized elderly. It was developed in three phases: 1) the development of the instrument; 2) the validation of the instrument contents; 3 ) clinical testing with elderly. In the first phase, the literature revision and development of the initial version of the instrument were accomplished. The second phase was carried out in two stages: $1^{\text {st }}$ ) initial version of the instrument to validate the contents of the items; $2^{\text {nd }}$ ) the development of the definitive version of the instrument. In the last phase, the final instrument was tested in ten elderly patients. It is expected that the use of this instrument by nurses would result in a better orientation to the older people, their family, their caregivers and the nursing team when they access the affected necessities in order to minimize the established difficulties.

KEYWORDS: Aged; Validation studies; Nursing methodology research.

\section{CONSTRUYENDO UN HISTÓRICO DE ENFERMERÍA PARA SER UTILIZADO CON EL ANCIANO INTERNADO EN UN HOSPITAL UNIVERSITARIO}

RESUMEN: Estudio metodológico objetivando construir un instrumento de recopilación de datos para el anciano hospitalizado. Se desarrolló en tres etapas: 1) la construcción del instrumento, 2) la validación del contenido del instrumento, 3) ensayos clínicos con ancianos. En la primera fase se llevaron a cabo la revisión de la literatura y la construcción de la versión original del instrumento, que condujeron a la identificación de 525 señales y síntomas, ordenados en una Escala Likert. La segunda fase, se celebró en dos etapas: $1^{\text {a }}$ ) versión inicial del instrumento encaminado a 21 enfermeras, para validar el contenido de los temas, identificándose 223 temas que presentaron la media ponderada e” a 80\%; 2ª) Construcción definitiva del instrumento constituido de siete partes. En la última fase fue testado el instrumento final en diez ancianos. Se espera que su utilización contribuya para que las enfermeras puedan servir de guía al anciano, la familia, cuidadores y al equipo de enfermería en la atención de las necesidades afectadas y en la minimización de las dificultades instaladas.

PALABRAS CLAVE: Anciano; Estudios de validación; Investigación metodológica en enfermería

\footnotetext{
*Trabalho extraído da dissertação de mestrado "Construção e testagem clínica de instrumento de coleta de dados para o idoso internado em um hospital universitário”. Programa de Pós-Graduação em Enfermagem/Mestrado em Enfermagem da Fundação Universidade Federal do Rio Grande-FURG.

${ }^{1}$ Enfermeira. Mestre em Enfermagem pela FURG. Especialista em Projetos Assistenciais de Enfermagem. Enfermeira assistencial do Hospital Universitário Dr. Miguel Riet Corrêa Jr da FURG. Membro do Grupo de Estudo e Pesquisa em Gerontogeriatria, Enfermagem/ Saúde e Educação-GEP-GERON/FURG/CNPq.

${ }^{2}$ Enfermeira. Doutora em Enfermagem pela UFSC. Professora do Departamento de Enfermagem e do Mestrado em Enfermagem da FURG. Orientadora da dissertação. Líder do GEP-GERON/FURG/CNPq.
}

Autor correspondente:

Fernanda Lucas Lopes

Rua: Herval do Sul, 258 - 96208-040 - Rio Grande-RS.

Recebido: 29/09/08

E-mail: fernanda.lopes@vetorial.net

Aprovado:24/03/08

Cogitare Enferm 2009 Jan/Mar; 14(1):22-9 


\section{INTRODUÇÃO}

O envelhecimento populacional vem se tornando um fenômeno global e, conseqüentemente, fonte de preocupação de profissionais e estudiosos de várias áreas de conhecimento, de órgãos governamentais e não-governamentais e da sociedade civil.

Projeções realizadas para o período de 75 anos, compreendido entre 1950 a 2025, indicam uma taxa de crescimento da população idosa da ordem de $1514,3 \%$, demonstrando que, enquanto a população em geral aumentará cinco vezes, o grupo etário, a partir de 60 anos, se tornará quinze vezes maior ${ }^{(1)}$.

No estado do Rio Grande do Sul, o aumento da população idosa é marcante, pois se verificam melhores condições sociais, sanitárias, econômicas e culturais nesse Estado. Fato que contribui para o aumento da expectativa de vida, atualmente em torno de 74 anos $^{(2)}$. No município de Rio Grande, no Extremo Sul do país, local de realização deste estudo, no ano de 2000, os idosos já representavam uma grande parcela da população, totalizando 20794 pessoas, pouco mais de $10 \%$ da população municipal total ${ }^{(3)}$, dados acima da média nacional que fica em torno de $9 \%$ da população total.

Um envelhecimento bem sucedido dependerá de alguns fatores do próprio indivíduo e, depois, da sociedade cujo dever é propiciar espaços, no qual resida o cuidado pelo outro, sem superproteção ou subestimação, promovendo laços sócio-afetivos capazes de integrá-los ou mantê-los nos grupos sociais $^{(4)}$. Aqui, destacamos o papel da enfermagem como profissão comprometida com o cuidado do ser humano em todo o processo de viver e morrer, incluindo a velhice, fase ainda tão cheia de preconceitos e tabus.

$\mathrm{Na}$ enfermagem, o cuidado significa um trabalho profissional específico. O planejamento da assistência de enfermagem é um dos meios que a enfermeira dispõe para aplicar os seus conhecimentos técnicos, científicos e humanos no cuidado ao ser humano saudável ou doente, além de caracterizar sua prática profissional, colaborando na definição de seu papel. Ao planejar a assistência de enfermagem estamos utilizando uma metodologia para sistematizar o cuidado e organizar as condições necessárias para sua execução ${ }^{(5-7)}$.

A Enfermagem ainda é uma profissão com déficit em um cuidado especializado ou pelo menos mais direcionado aos idosos e, a Sistematização da Assistência de Enfermagem (SAE) tem se mostrado uma das principais ferramentas metodológicas para o desempenho sistemático de uma prática assistencial especializada e de qualidade ${ }^{(8)}$.

O cuidado de enfermagem direcionado ao idoso requer, da enfermeira, conhecimento científico e uma visão global das necessidades específicas de cada indivíduo ${ }^{(9)}$. A SAE possibilitará à enfermeira desenvolvimento e aprimoramento do cuidado ao idoso, pois gera intervenções de enfermagem que tornam possível um cuidado de qualidade. Com a SAE, a relação entre enfermeira e ser humano idoso poderá tornar-se mais estreita. Existe um maior envolvimento da profissional, pela necessidade de sua presença mais efetiva ao lado dele. Para a realização do Histórico de Enfermagem, é preciso diálogos e visitas freqüentes, na busca da constatação de diagnósticos, para que ações sejam planejadas e as intervenções realizadas e avaliadas, a cada momento em que se façam necessárias.

Considerando o número cada vez mais crescente de idosos atendidos em hospitais universitários, bem como a necessidade de entendimento de que essa população apresenta especificidades e multidimensionalidade que precisam ser postas em relevância no direcionamento do cuidado para com eles, é que se originou este estudo. Necessita-se perceber o cuidado ao idoso de forma sistematizada e, para tanto, torna-se necessária a elaboração de um instrumento de coleta de dados, um formulário para Histórico de Enfermagem, que melhor abarque suas necessidades e melhor direcione a identificação dos Diagnósticos de Enfermagem, para assim, podermos, mais adiante, estabelecer toda a SAE para esses idosos internados.

Acredita-se que a utilização de um Histórico de Enfermagem se tornará um caminho para a implantação da SAE que pode levar ao despertar da enfermeira sobre a importância do seu fazer profissional, destacando a Enfermagem como disciplina científica. Assim, este estudo teve como objetivo: construir um instrumento para coleta de dados, Histórico de Enfermagem, a ser utilizado no atendimento ao idoso internado em um hospital universitário.

\section{CAMINHOS DA METODOLOGIA}

Nesse estudo foi utilizada a pesquisa metodológica, que investiga os métodos de obtenção, organização e análise dos dados por meio da 
elaboração, validação e avaliação de instrumentos e técnicas de pesquisa. Nela o pesquisador concentrase em elaborar um instrumento que seja capaz de medir, de maneira precisa, a satisfação das necessidades dos clientes sob seus cuidados. Seu objetivo é elaborar um instrumento confiável, preciso e utilizável, o que pode ser utilizado por outros pesquisadores $^{(10-11)}$.

Participaram deste estudo 16 sujeitos: 12 enfermeira assistenciais e quatro decentes. Foi utilizado como critério de inclusão para participar da pesquisa: para enfermeiras assistenciais atuar na instituição pesquisada há mais de um ano e prestar cuidado ao idoso e para enfermeiras docentes atuar há mais de um ano, no ensino da Gerontologia, Enfermagem Fundamental, Saúde do Adulto (situação clínica e/ou cirúrgica).

Após a autorização da instituição para realização do estudo, o projeto de pesquisa foi encaminhado ao Comitê de Ética e Pesquisa da Área de Saúde (CEPAS), da FURG. Após a aprovação do projeto pelo CEPAS, sob $n^{\circ}$ do processo $038 / 2006$, foi iniciada a coleta de dados, respeitando os aspectos éticos, por meio da elaboração dos Termos de Consentimento Livre e Esclarecido (TCLE), nos quais os sujeitos da pesquisa foram informados sobre a finalidade da pesquisa, do sigilo quanto a sua identidade, da privacidade quanto as suas informações e da liberdade de deixar de participar do estudo sem nenhum constrangimento ou prejuízo, em qualquer etapa. O TCLE direcionado ao idoso apresentou uma linguagem mais simples e letras maiores, tamanho 14, para facilitar a sua leitura ou a leitura por seu familiar/ cuidador, assim foram respeitados os aspectos éticos e a Resolução nº 196/96 que regulamenta as diretrizes e normas de pesquisa envolvendo seres humanos ${ }^{(13)}$.

Este estudo foi constituído de três fases: $1^{\mathrm{a}}$ ) construção do instrumento; $2^{\mathrm{a}}$ ) validação de conteúdo do instrumento construído com enfermeiras assistenciais e docentes, da gerontologia e áreas afins; $3^{a}$ ) testagem clínica do Histórico de Enfermagem nos idosos internados em uma clínica médica.

\section{RESULTADOS}

Construção do Instrumento Inicial (Escala Likert)

Desenvolveu-se um instrumento contemplando as Necessidades Humanas Básicas (NHB) de $\operatorname{Horta}^{(12)}$, com suas definições e manifestações:
1. Necessidades Psicobiológicas: oxigenação; hidratação; nutrição; eliminação; sono e repouso; exercício e atividade física; sexualidade; abrigo; mecânica corporal; mobilidade; cuidado corporal; integridade cutâneo-mucosa; integridade física; regulação: térmica, hormonal, neurológica, hidrossalina, eletrolítica, imunológica, crescimento celular, vascular; locomoção; percepção: olfativa, visual, auditiva, tátil, gustativa, dolorosa; ambiente e terapêutica.

2. Necessidades Psicossociais: segurança; amor; liberdade; comunicação; criatividade; aprendizagem; gregária; recreação; lazer; espaço; orientação no tempo e no espaço; aceitação; autorealização; auto-estima; participação; auto-imagem e atenção.

3. Necessidades Psicoespirituais: religiosa ou teológica, ética ou de filosofia de vida.

Essas necessidades foram apresentadas em uma Escala Likert com itens numerados de um a cinco, utilizados para medir o grau das manifestações das necessidades importantes para construir outro instrumento que direcionou à avaliação de enfermagem no atendimento ao idoso internado em uma unidade hospitalar. Para os itens desta escala foram designados os seguintes valores: $1=$ nunca necessário; $2=$ raramente necessário; 3= algumas vezes necessário; $4=$ muitas vezes necessário; $5=$ sempre necessário.

Esse instrumento foi entregue para dois enfermeiros docentes, doutores, com vivência em cuidado ao idoso, para que fosse realizada uma préavaliação. A idéia era retirar os termos repetidos e desconexos em relação ao tema. Após essa avaliação, o instrumento ficou constituído por 17 páginas, contendo na primeira parte uma Escala Likert, apresentando as 36 NHB, suas respectivas definições, e 525 manifestações dessas necessidades afetadas no idoso; e, na segunda parte, os dados sócio-demográficos desses idosos.

\section{Validação de conteúdo do instrumento construído com enfermeiras assistenciais e docentes, da gerontologia e áreas afins}

O objetivo foi realizar, com as enfermeiras docentes e assistenciais, a validação de conteúdo do instrumento de coleta de dados para o atendimento do idoso internado em uma unidade de internação hospitalar. Esta segunda fase ocorreu em duas etapas.

$1^{\text {a }}$ etapa: realizada nos meses de agosto e setembro de 2006 quando a Escala Likert, foi entregue 
a um grupo de 21 enfermeiras, das quais 16 fizeram a devolução. Foi solicitado das enfermeiras que, levando em consideração o atendimento das NHB do idoso, opinassem, marcando um (X) na alternativa do instrumento que representasse maior importância para $o$ atendimento das NHB do idoso.

De posse dos 16 instrumentos devolvidos, foi realizada a tabulação dos dados em uma planilha eletrônica, por meio do Excel, sendo calculada a média ponderada dos valores determinados pelo grupo para cada um dos itens do instrumento, considerando-se para esse cálculo os seguintes pesos: $1=0 ; 2=0,25$; 3 $=0,50 ; 4=0,75$ e $5=1$. Foram descartados todos os itens com média ponderada $<0,79$. Os itens com média ponderada $>=0,80$ foram utilizados para construir 0 instrumento de coleta de dados para o atendimento ao idoso.

Após a realização do cálculo descrito, para cada item do instrumento, foram excluídas das 36 NHB apresentadas por $\mathrm{Horta}^{(12)}$, pelo fato de suas manifestações não terem alcançado média ponderada $>=0,80$, sendo elas: regulação eletrolítica, crescimento celular, criatividade, recreação/lazer, auto-realização, atenção, religiosa ou teológica ou de filosofia de vida, restando 33 necessidades com 223 manifestações.

$2^{\text {a }}$ etapa: foi realizada nos mês de outubro de 2006, com a construção do Histórico de Enfermagem e o envio para avaliação do conteúdo e da forma do instrumento pelo mesmo grupo das 16 enfermeiras.

Para a construção do Histórico de Enfermagem lançou-se mão da literatura especializada na área de enfermagem gerontogeriátrica e de acervos da gerontologia e da geriatria. A grande maioria dos instrumentos que são utilizados para realizar a avaliação do idoso inclui os seguintes aspectos: caracterização geral do idoso, história clínica, revisão geral dos sistemas, antecedentes patológicos, antecedentes familiares, hábitos e vícios, sono e repouso, exame físico, avaliação do estado mental com miniexame do estado mental, escala de depressão, escala de atividades física e instrumental da vida diária e espaço para anotações e impressões do entrevistador.

Optou-se pela adoção dos mesmos critérios para a construção do Histórico para o atendimento ao idoso, nos quais foram distribuídas as 223 manifestações das NHB que alcançaram um índice da média ponderada $>=0,80$. O instrumento de coleta de dados para o atendimento ao idoso ficou constituído de sete partes: 1) Identificação, 2) Condições gerais, 3) Avaliação funcional, incluindo as atividades da vida diária básicas, instrumentais e avançadas, 4) Avaliação cognitiva, 5) Avaliação sócio-familiar, 6) Avaliação das necessidades humanas básicas; 7) Outras informações de interesse da enfermeira.

\section{Perfil sócio-demográfico dos participantes da primeira etapa da pesquisa}

Participaram dessa etapa, conforme as características sócio-demográficas, 13 mulheres e três homens. A maior freqüência de idade foi de 20 a 30 anos ou 41 a 50 anos de idade, mostrando a heterogeneidade etária (grupo muito jovem e grupo mais maduro) dos investigados. Na pós-graduação em Enfermagem, prevaleceu a especialização. Em experiência profissional, acompanhando o grupo etário, foram mais freqüentes entre 1 a 5 anos e entre 16 a 20 anos. Já na experiência vivenciada no cuidado ao idoso foi mais freqüente de 1 a 5 anos, mostrando que é bem recente o aumento de pessoas idosas internadas em hospitais. Quanto à posição na Enfermagem, 12 enfermeiras exercem suas funções na assistência e quatro, na docência.

\section{Descrição do Instrumento de Coleta de Dados para $o$ atendimento ao idoso}

A primeira parte do instrumento corresponde à Identificação, para a coleta dos dados que irão auxiliar a enfermeira a caracterizar o idoso, constituída dos itens: nome, idade, sexo, escolaridade, condição civil, profissão/ocupação, religião e endereço. Lembramos que esse item não contém nenhuma das manifestações de necessidades identificadas como importantes pelo grupo de enfermeiras para fazer parte do instrumento.

A segunda parte do instrumento Condições gerais, constituiu-se de: pressão arterial, temperatura axilar, freqüência cardíaca, freqüência respiratórias, Doenças Crônicas Não-Transmissíveis (DCNT) e outras que foram apresentadas como manifestações das necessidades de oxigenação; regulação térmica, hidrossalina e vascular; aprendizagem (educação à saúde).

A terceira parte ficou constituída pela Avaliação funcional, na qual é avaliada a capacidade de realizar as Atividades da Vida Diária (básicas, instrumentais e avançadas) e, também, são incluídas as manifestações das necessidades: nutrição; eliminação; mecânica corporal; exercícios e atividades físicas; motilidade; cuidado corporal; 
locomoção; liberdade; gregária; ambiente.

A avaliação funcional é um procedimento essencial para o cuidado ao idoso, contribuindo para o alcance de objetivos como: contribuir para que a enfermeira identifique e utilize os recursos adequados; utilizar uma abordagem sistemática capaz de avaliar os déficits das pessoas idosas que, muitas vezes, acabam passando despercebidos; proporcionar condições para avaliação do progresso e deterioração que possa acontecer com o passar do tempo e subsidiar meios para que a enfermeira avalie a capacidade do idoso de viver só e com segurança( ${ }^{(14)}$.

No instrumento, é necessário determinar o nível de dependência para realizar as atividades básicas e instrumentais, que pode ser: 1- quando o idoso realiza a atividade sozinho; 2- quando ele precisa de ajuda e, 3- quando ele não consegue realizar e necessita que alguém faça por ele.

Nas atividades da vida diária básicas avaliamse as capacidades para se alimentar, banhar, vestir, higienizar, controlar a eliminação de urina e fezes, deambular e locomover. Nas atividades instrumentais da vida diária é avaliada a capacidade do idoso para fazer compras, preparar comida, cuidar da casa, usar meios de transporte, tomar sua medicação e cuidar de suas finanças. Nas atividades da vida diária avançadas foi necessário marcar a alternativa que correspondia à resposta do idoso, no que dizia respeito à participação em grupos sociais e religiosos, como também preenchimento do seu tempo livre e descrição do tipo, local e freqüência das atividades de lazer e de recreação, caso respondam $\operatorname{sim}^{(11)}$.

A quarta parte do instrumento dizia respeito à Avaliação cognitiva, em que foram incluídas as manifestações das necessidades de: regulação neurológica; orientação no tempo e espaço; aprendizagem.

O objetivo da avaliação cognitiva é detectar alterações na função mental, da memória, da percepção, da comunicação, da compreensão de cálculos, da orientação, da resolução de problemas, da linguagem dos processos de pensamento e outros $^{(14)}$. As demências, incluindo a Doença de Alzheimer e as depressões são algumas das doenças comuns e que mais agravam o estado de saúde mental durante a velhice.

Nesse instrumento, a avaliação da função cognitiva ficou constituída das partes: memória - dia de hoje, dia da semana, idade, dia/mês/ano do nascimento; orientação - local onde se encontra e endereço; aprendizagem - conhecimento do seu estado de saúde; Informações sobre fatos cotidianos - nome do presidente do Brasil e nome do prefeito de sua cidade; capacidade de cálculo - contar de três em três e realizar cálculos simples.

A quinta parte do instrumento contém a Avaliação da situação sócio-familiar, em que foram incluídas as manifestações das necessidades de: abrigo; segurança; gregária e liberdade, que ficou composta dos seguintes itens: com quem o idoso reside, quantas pessoas vivem com ele, quem são essas pessoas, se existe um responsável pelo cuidado, se existe um bom relacionamento no lar, se depende dos familiares e amigos e se deseja ou não receber visitas.

A sexta parte do instrumento foi constituída pela Avaliação das necessidades humanas básicas, e incluídas manifestações das necessidades de: oxigenação; nutrição; hidratação; eliminação; sono e repouso; exercícios; atividade física; sexualidade; integridade cutâneo-mucosa; integridade física; imunológicas; percepção; abrigo; segurança; espaço; amor; auto-estima; auto-imagem.

Na avaliação da necessidade de oxigenação, incluíram-se os itens: dispnéia, obstrução das vias respiratórias e cianose. Na necessidade de nutrição/ hidratação, foram incluídos os itens: peso; altura; anorexia; diminuição do apetite; alterações na mastigação e deglutição; presença de sonda para alimentação; alterações no turgor cutâneo e edema. Na necessidade de eliminação, foram incluídos: hábitos intestinais e urinários, anotando a freqüência e características; anúria; oligúria; constipação e dificuldade e esforço para evacuar.

Na necessidade de sono e repouso/exercícios e atividade física: hábitos de sono; problemas para adormecer; uso de sedativos; insônia; se apresenta incapacidade moderada para atividade e qual; se apresenta incapacidade grave para a atividade e qual; se apresenta atividade motora diminuída, se apresenta dor ao movimento; inatividade; fadiga; se movimenta adequadamente todas as partes do corpo; partes do corpo que não movimenta bem; coordenação prejudicada; incapacidade para deambular, levantar-se ou sair do leito; alterações na postura; alterações no alinhamento da coluna; distúrbios na marcha; presença de hemiplegia, paraplegia e paralisia; risco para quedas e se necessita de ajuda para deambular/deslocar-se. Na necessidade de sexualidade: isolamento e uso de medicamentos que afetam a libido, sendo os principais os anti-hipertensivos, tranqüilizantes e anti-depressivos. 
Na integridade cutâneo-mucosa/integridade física: pele hidratada; pele fria; pela pálida; pele hiperemiada; edema; prurido; cianose periférica, presença de lesões/ferimentos, anotando tipo e local; presença de escaras, anotando local e estágio ou grau. Na necessidade imunológica: alergias e suscetibilidade às infecções. Na percepção, foram incluídos os itens: visão (perda da visão, secreções oculares, dificuldades para enxergar, uso de óculos, uso de lentes); audição (surdez, utilização de prótese, zumbido, secreções, dificuldade de comunicação/pronunciar palavras); paladar (preservado, diminuído, ausente); tato (sensibilidade tátil, sensibilidade à dor, apresenta dor).

Na última parte do instrumento denominada de Outras informações de interesse da enfermeira, ficou reservado um espaço para anotações de outros dados de interesse da enfermeira, que podem ser incluídas manifestações de NHB observadas no idoso e não contempladas no instrumento.

O instrumento descrito foi enviado aos 16 enfermeiros que participaram da primeira etapa deste estudo, solicitando-lhes que lessem atentamente e dessem suas opiniões no que diz respeito à forma e ao conteúdo, desses, dez foram devolvidos.

\section{Avaliação do histórico de enfermagem pelas enfermeiras}

No que se refere à forma e ao conteúdo do instrumento, as enfermeiras foram unânimes em afirmar que o mesmo apresentava-se objetivo e aplicável ao atendimento ao idoso internado em um hospital, além das observações para alguns itens foram apresentadas algumas sugestões. Tais informações e sugestões ficaram explícitas nos comentários das enfermeiras, que passamos a relatar.

Quanto à avaliação da forma, elas afirmaram que o mesmo é objetivo, prático, conciso, de fácil compreensão e utilização, de acordo com uma seqüência lógica das informações. Quanto à avaliação de conteúdo, no item condições gerais, as enfermeiras relataram que possui informações suficientes para identificar o idoso, individualizando-o durante a coleta de dados. Afirmaram, ainda, que o instrumento possui dados básicos para a identificação do idoso. Foi sugerido acrescentar a unidade, o número da enfermaria e o leito do idoso.

Em relação às condições gerais, relataram que os dados contemplados são importantes para a Enfermagem e para a avaliação do estado do idoso (se regular, estável ou grave), além de destacarem a importância do espaço para as Doenças Crônicas NãoTransmissíveis (DCNTs), tornando-se possível manter um plano de cuidados de acordo com as patologias. Afirmaram que ficou rápido e prático preenchê-lo, dando uma sinalização acerca de eventuais agravos crônicos que possam afetar a qualidade de vida do idoso. Foi sugerido excluir nesta parte os sinais vitais porque já existe, no leito, uma planilha de controle. No entanto, acredita-se que a verificação dos sinais vitais faz parte de um bom e completo exame físico, por esse motivo optou-se por mantê-los.

Afirmaram que a avaliação funcional contempla todos os itens, apresentando qualidade no objetivo final. Relataram que está bem direcionada, com praticidade na forma de coleta, além de fornecer informações de como o idoso se relaciona fora da instituição hospitalar. Elas acreditaram que a avaliação funcional seja necessária para que a Enfermagem saiba se o idoso necessita de ajuda e que tipo de ajuda.

$\mathrm{Na}$ avaliação cognitiva os dados foram avaliados como pertinentes, porém foi salientado que a enfermeira necessita ter o bom senso de situar o contexto geográfico e cultural do idoso, pois com freqüência são recebidos idosos de zonas urbanas e rurais ou com baixo nível educacional. Na avaliação sócio-familiar, destacaram que é pertinente, para avaliar a presença de eventuais conflitos familiares, a omissão no acompanhamento do idoso e o preenchimento de endereços inexistentes.

No item destinado às impressões $\mathrm{da}(\mathrm{o})$ enfermeira(o), foi julgado importante um local para que possa ser feita essa avaliação pessoal e subjetiva da enfermeira, além de oportunizar o registro de dados não contemplados no formulário.

Entre outras sugestões/solicitações, foi pedido o acréscimo de um item que contemplasse: a viuvez; as condições circulatórias, relacionada com edema de membros inferiores; a sexualidade, por ter ficado muito restrita; nas condições imunológicas o estado vacinal; na percepção do paladar o uso de prótese dentária. Percebeu-se a falta de alguns desses itens, mas foi preciso respeitar a opinião das enfermeiras que não consideraram nenhuma das manifestações dessas necessidades como importantes para serem incluídas no instrumento, ou seja, tais itens não alcançaram à média ponderada estabelecida na metodologia e, por isso não foram contemplados no Histórico de Enfermagem. Para este caso, ou similares, cabe lembrar que existe um espaço para que a enfermeira 
registre dados importantes, que não estejam contemplados no roteiro deste instrumento. A partir dos resultados dessa etapa da pesquisa, foi realizada uma revisão no instrumento de coleta de dados, sendo acrescentado às sugestões apresentadas, o que resultou em sua versão final.

\section{Testagem clínica do histórico de enfermagem nos idosos internados em uma clínica médica}

Constituiu-se na aplicação clínica do Histórico de Enfermagem, realizada com dez idosos de uma unidade de clínica médica de um hospital universitário, tendo como objetivo verificar a adequação do instrumento na prática. Nessa fase, foram anotadas as dificuldades que surgiram durante a aplicação do instrumento, como também, o tempo gasto na sua aplicação.

Com a aplicação, na prática, desse instrumento, foi possível confirmar as opiniões das enfermeiras a respeito dele, pois apresenta conteúdo claro, é de fácil compreensão, não permite respostas dúbias. Em média, foram gastos 20 minutos para o preenchimento do mesmo, não sendo observado nem relatado incômodo por parte dos idosos.

Apesar de saber que o objetivo dessa fase era verificar a adequação do instrumento à prática foi possível observar que, através dos dados coletados, podem-se obter a identificação de Diagnósticos de Enfermagem para posterior implantação de um Plano de Cuidados. Tal fato confirma que esse Histórico de Enfermagem pode ser um facilitador para uma posterior implantação da SAE não apenas nessa unidade de internação como em toda a instituição.

\section{CONSIDERAÇÕES FINAIS}

Atingiram-se os objetivos iniciais desta pesquisa, pois foi realizada a construção de um instrumento de coleta de dados com o idoso internado em um hospital universitário. Além disso, foi possível realizar, com as enfermeiras assistenciais e docentes, a validação inicial e final do conteúdo e, também, realizar a testagem clínica do instrumento com os idosos internados numa unidade de clínica médica de um hospital universitário.

No decorrer do processo de envelhecimento, o grau de dependência tende a aumentar, exigindo um cuidado apropriado e eficaz, direcionado para o atendimento das necessidades individuais e coletivas, e às particularidades da idade. Este trabalho permitiu- nos o conhecimento e compreensão da importância do atendimento das NHB direcionadas ao cuidado do idoso, mostrando que o Histórico de Enfermagem torna-se uma importante ferramenta para o desenvolvimento da SAE nesta instituição, principalmente no que diz respeito as suas duas primeiras fases: Levantamento de dados e identificação de Diagnósticos de Enfermagem.

A construção do instrumento além de ser um estudo pertinente, tendo em vista a população idosa brasileira representar a que mais cresce e a que mais procura os serviços de saúde, vem preencher uma lacuna existente no hospital pesquisado, no qual não existe um instrumento específico para registrar os dados coletados pela enfermeira durante $\mathrm{o}$ atendimento ao idoso.

A falta ou a utilização inadequada do Histórico de Enfermagem direcionado ao idoso hospitalizado, bem como o não conhecimento de suas NHB afetadas, pode dificultar o cuidado, pois muitas das alterações decorrentes do processo de envelhecimento podem deixar de ser identificadas. O planejamento e a implementação de ações adequadas ao idoso, podem contribuir para a redução do tempo de internação hospitalar, além de diminuir o número de reinternações hospitalares.

Com o aumento da população idosa, faz-se necessário que os profissionais da área de saúde, inclusive as enfermeiras, conheçam as fases do processo de envelhecimento, normal e patológico, tornando-as aptas a prestar um cuidado voltado às especificidades e multidimensionalidade dessa população. Tal afirmativa remete para a importância de estimular cada vez mais a inserção da disciplina de gerontogeriatria nos currículos dos cursos de graduação em Enfermagem.

A partir dos resultados deste estudo, pode-se afirmar que na utilização desse instrumento de coleta de dados, de forma segura e comprometida, a enfermeira obtém subsídios relevantes para o cuidado do idoso, sua família e seus cuidadores. No contexto hospitalar, observa-se com freqüência, idosos isolados, tristes, pouco comunicativos sem verbalizar suas queixas, inquietações e medos. Assim, este histórico representará uma oportunidade para a enfermeira amenizar o desconforto e criar uma relação e um ambiente de confiança, que possibilite ao idoso expressar seus sentimentos e as suas queixas, tornando a hospitalização mais amena.

Para o ensino e para a pesquisa, este trabalho 
pretende representar mais uma opção de modelo para ser aplicado, testado e fomentado nas discussões dos variados cenários de prática e teoria. A aplicação e a utilização deste instrumento representará, para o grupo de enfermeiras, assistenciais e docentes, um avanço na qualidade do cuidado ao idoso, pois irá trabalhar com aspectos específicos dessa população de maneira organizada e individualizada, contribuindo para uma velhice mais digna e saudável.

Espera-se, ainda, que este trabalho possa contribuir para que as chefias dos Serviços de Enfermagem procurem facilitar a participação das enfermeiras, em cursos de atualização e pósgraduação que contemplem a instrumentalização para a SAE. E mais que isso, estimule a instrumentalização dessas profissionais para o cuidado ao idoso, tendo em vista a carência de conteúdo sobre o processo de envelhecimento e alterações decorrentes desse processo nos cursos de graduação.

\section{REFERÊNCIAS}

1. Veras R. País jovem com cabelos brancos - a saúde do idoso no Brasil. Rio de Janeiro: Relumé-Dumará; 1995.

2. Rio Grande do Sul. Conselho Estadual do Idoso. Os idosos do Rio Grande do Sul: um estudo multidimensional de suas condições de vida - Relatório de pesquisa. Porto Alegre: Conselho Estadual do Idoso; 1997.

3. IBGE. Instituto Brasileiro de Geografia e Estatística [base de dados na Internet]. Brasília: Ministério do Planejamento, Orçamento e Gestão. 2000. [ acesso em: 2005 Abr 10]. Disponível em: www.ibge.gov.br/home/ estatistica/populacao/perfilidosohtm.

4. Gonçalves LHT, Vahl EAC, Borges IC. O idoso contador de histórias - a autopromoção de um envelhecimento criativamente bem sucedido. Texto Contexto Enferm. 2001 Mai/Ago; 10(2):94-115.

5. Garcia TR. Modelos metodológicos para validação de diagnósticos de enfermagem. Acta Paul Enferm. 1998 Set/Dez; 11(3): 24-31.

6. Kubo CH, Ribeiro PJ, Aguiar LAK, Toledo CF, Barros SMO, Borges DR. Construção e implementação de ações de enfermagem em ambulatório de gastroenterologia. Rev Latino-Am Enferm. 2003 Nov/Dez; 11(6): 816-22.

7. Lopes FL, Szewczyk MSC, Lunardi VL. Santos SSC. SAE como um novo fazer na atividade cuidativa da enfermagem com base na complexidade de Edgar Morin . Cogitare Enferm. 2007 Jan/Mar; 12(1): 115-19.

8. Araújo LA, Bachion MM. Diagnósticos de enfermagem do padrão mover em idosos de uma comunidade atendida pelo Programa de Saúde da Família. Rev Esc Enferm USP. 2005; 39(1):53-61.

9. Teixeira MB, Fernandes RAQ. Diagnósticos de enfermagem identificados em idosos com distúrbio mental. Rev Bras Enferm. 2003 Nov/Dez; 56(6): 619-23.

10. Polit DF, Hungler BP. Fundamentos de pesquisa em enfermagem. Porto Alegre: Artes Médicas; 1999.

11. Porto MLL. Construção e validação de um instrumento de coleta de dados para o idoso no Programa de Saúde da Família [dissertação]. João Pessoa (PB): Centro de Ciências da Saúde, Universidade Federal da Paraíba; 2004.

12. Horta W. Processo de Enfermagem. São Paulo (SP): EPU; 1979.

13. Ministério da saúde (BR). Conselho Nacional de Saúde. Diretrizes e normas regulamentadoras de pesquisa envolvendo seres humanos. Resolução n. 196, 10 de outubro de 1996. Brasília; 1996.

14. Nettina SM. Prática de Enfermagem. 6 aed. Rio de Janeiro: Gunabara Koogan; 1998. 